\title{
Generalized Löb’s Theorem. Strong Reflection Principles and Large Cardinal Axioms
}

\author{
J. Foukzon ${ }^{1}$, E. R. Men'kova ${ }^{2}$ \\ ${ }^{1}$ Israel Institute of Technology, Haifa, Israel \\ ${ }^{2}$ Lomonosov Moscow State University, Moscow, Russia \\ Email: jaykovfoukzon@list.ru,E_Menkova@mail.ru \\ Received February 9, 2013; revised March 13, 2013; accepted April 14, 2013
}

Copyright (C) 2013 J. Foukzon, E. R. Men'kova. This is an open access article distributed under the Creative Commons Attribution License, which permits unrestricted use, distribution, and reproduction in any medium, provided the original work is properly cited.

\begin{abstract}
In this article, a possible generalization of the Löb's theorem is considered. Main result is: let $\kappa$ be an inaccessible cardinal, then $\neg \operatorname{Con}(Z F C+\exists \kappa)$.
\end{abstract}

Keywords: Löb's Theorem; Second Godel Theorem; Consistency; Formal System; Uniform Reflection Principles; $\omega$-Model of ZFC; Standard Model of ZFC; Inaccessible Cardinal

\section{Introduction}

Let $T h$ be some fixed, but unspecified, consistent formal theory.

Theorem 1 [1]. (Löb's Theorem).

If $T h \vdash \exists x \operatorname{Prov}_{T h}(x, \breve{n}) \rightarrow \phi_{n}$ where $x$ is the Gödel number of the proof of the formula with Gödel number $n$, and $\breve{n}$ is the numeral of the Gödel number of the formula $\varphi_{n}$, then $T h \vdash \phi_{n}$. Taking into account the second Gödel theorem it is easy to be able to prove

$\exists x \operatorname{Prov}_{T h}(x, \breve{n}) \rightarrow \varphi_{n}$, for disprovable (refutable) and undecidable formulas $\varphi_{n}$. Thus summarized, Löb's theorem says that for refutable or undecidable formula $\varphi$, the intuition "if exists proof of $\varphi$ then $\varphi$ " is fails.

Definition 1. Let $M_{\omega}^{T h}$ be an $\omega$-model of the $T h$. We said that, $T h^{\#}$ is a nice theory over $T h$ or a nice extension of the $T h$ iff:

1) $T h^{\#}$ contains $T h$;

2) Let $\Phi$ be any closed formula, then

$$
\left[\operatorname{Th} \vdash \operatorname{Pr}_{T h}\left([\Phi]^{c}\right)\right] \&\left[M_{\omega}^{T h} \vDash \Phi\right]
$$

implies $T h^{\#} \vdash \Phi$.

Definition 2. We said that, $T h^{\#}$ is a maximally nice theory over $T h$ or a maximally nice extension of the $T h$ iff $T h^{\#}$ is consistent and for any consistent nice extension $T h^{\prime}$ of the $T h: \operatorname{Ded}\left(T h^{\#}\right) \subseteq \operatorname{Ded}\left(T h^{\prime}\right)$ implies

$\operatorname{Ded}\left(T h^{\#}\right)=\operatorname{Ded}\left(T h^{\prime}\right)$.

Theorem 2. (Generalized Löb's Theorem). Assume that 1) $\operatorname{Con}(T h)$ and 2) $T h$ has an $\omega$-model $M_{\omega}^{T h}$. Then theory $T h$ can be extended to a maximally consistent nice theory $T h^{\#}$.

\section{Preliminaries}

Let $T h$ be some fixed, but unspecified, consistent formal theory. For later convenience, we assume that the encoding is done in some fixed formal theory $S$ and that $T h$ contains $S$. We do not specify $S$ - it is usually taken to be a formal system of arithmetic, although a weak set theory is often more convenient. The sense in which $S$ is contained in $T h$ is better exemplified than explained: If $S$ is a formal system of arithmetic and $T h$ is, say, $Z F C$, then $T h$ contains $S$ in the sense that there is a well-known embedding, or interpretation, of $S$ in $T h$. Since encoding is to take place in $S$, it will have to have a large supply of constants and closed terms to be used as codes. (e.g. in formal arithmetic, one has $\overline{0}, \overline{1}, \cdots) S$ will also have certain function symbols to be described shortly. To each formula, $\Phi$, of the language of $T h$ is assigned a closed term, $[\Phi]^{c}$, called the code of $\Phi$. [N. B. If $\Phi(x)$ is a formula with a free variable $x$, then $[\Phi(x)]^{c}$ is a closed term encoding the formula $\Phi(x)$ with $x$ viewed as a syntactic object and not as a parameter.] Corresponding to the logical connectives and quantifiers are function symbols, $\operatorname{neg}(\cdot), \operatorname{imp}(\cdot)$, etc., such that, for all formulae $\Phi, \Psi: S \mid-\operatorname{neg}\left([\Phi]^{c}\right)$

$=[\neg \Phi]^{c}, S \mid-\operatorname{imp}\left([\Phi]^{c},[\Psi]^{c}\right)=[\Phi \rightarrow \Psi]^{c}$ etc. 
Of particular importance is the substitution operator, represented by the function symbol $\operatorname{sub}(\cdot, \cdot)$. For formulae $\Phi(x)$, terms $t$ with codes $[t]^{c}$ :

$$
S \mid-\operatorname{sub}\left([\Phi(x)]^{c},[t]^{c}\right)=[\Phi(t)]^{c} .
$$

Iteration of the substitution operator $s u b$ allows one to define function symbols $\mathrm{sub}_{3}, \mathrm{sub}_{4}, \cdots, \mathrm{sub}_{n}$ such that

$$
\begin{aligned}
& S \mid-\operatorname{sub}_{n}\left(\left[\Phi\left(x_{1}, x_{2}, \cdots, x_{n}\right)\right]^{c},\left[t_{1}\right]^{c},\left[t_{2}\right]^{c}, \cdots,\left[t_{n}\right]^{c}\right) \\
& =\left[\Phi\left(t_{1}, t_{2}, \cdots, t_{n}\right)\right]^{c}
\end{aligned}
$$

It well known [2,3] that one can also encode derivations and have a binary relation $\operatorname{Prov}_{T h}(x, y)$ (read " $x$ proves $y$ " or " $x$ is a proof of $y$ ") such that for closed $t_{1}, t_{2}: S \mid-\operatorname{Prov}_{T h}\left(t_{1}, t_{2}\right)$ iff $t_{1}$ is the code of a derivation in $T h$ of the formula with code $t_{2}$. It follows that

$$
T h \vdash \Phi \leftrightarrow S \vdash \operatorname{Prov}_{T h}\left(t,[\Phi]^{c}\right)
$$

for some closed term $t$. Thus one can define predicate $\operatorname{Pr}_{T h}(y)$ :

$$
\operatorname{Pr}_{T h}(y) \leftrightarrow \exists x \operatorname{Prov}_{T h}(x, y),
$$

and therefore one obtain a predicate asserting provability.

Remark 2.1. We note that is not always the case that $[2,3]$ :

$$
T h \vdash \Phi i \leftrightarrow S \vdash \operatorname{Pr}_{T h}\left([\Phi]^{c}\right)
$$

It well known [3] that the above encoding can be carried out in such a way that the following important conditions $D 1, D 2$ and $D 3$ are met for all sentences [2,3]:

$$
\begin{aligned}
& \text { D1. Th } \vdash \Phi \text { implies } S \vdash \operatorname{Pr}_{T h}\left([\Phi]^{c}\right), \\
& \text { D2. } S \vdash \operatorname{Pr}_{T h}\left([\Phi]^{c}\right) \rightarrow \operatorname{Pr}_{T h}\left(\left[\operatorname{Pr}_{T h}\left([\Phi]^{c}\right)\right]^{c}\right), \\
& \text { D3. } S \vdash \operatorname{Pr}_{T h}\left([\Phi]^{c}\right) \wedge \operatorname{Pr}_{T h}\left([\Phi \rightarrow \Psi]^{c}\right) \\
& \rightarrow \operatorname{Pr}_{T h}\left([\Psi]^{c}\right) .
\end{aligned}
$$

Conditions $D 1, D 2$ and $D 3$ are called the Derivability Conditions.

Assumption 2.1. We assume now that:

1) the language of $T h$ consists of:

numerals $\overline{0}, \overline{1}, \cdots$

countable set of the numerical variables: $\left\{v_{0}, v_{1}, \cdots\right\}$ countable set $F$ of the set variables:

$$
F=\{x, y, z, X, Y, Z, \mathfrak{R}, \cdots\}
$$

countable set of the $n$-ary function symbols: $f_{0}^{n}, f_{1}^{n}, \ldots$ countable set of the $n$-ary relation symbols: $R_{0}^{n}, R_{1}^{n}, \cdots$ connectives: $\neg, \rightarrow$

quantifier: $\forall$.

2) $T h$ contains $Z F C$
3) $T h$ has an $\omega$-model $M_{\omega}^{T h}$.

Theorem 2.1. (Löb's Theorem). Let be 1) Con(Th) and 2) $\phi$ be closed. Then

$$
T h \vdash \operatorname{Pr}_{T h}\left([\phi]^{c}\right) \rightarrow \phi \text { iff } T h \vdash \phi .
$$

It well known that replacing the induction scheme in Peano arithmetic $\boldsymbol{P} \boldsymbol{A}$ by the $\omega$-rule with the meaning "if the formula $A(n)$ is provable for all $n$, then the formula $A(x)$ is provable":

$$
\frac{A(0), A(1), \cdots, A(n), \cdots}{\forall x A(x)},
$$

leads to complete and sound system $P A_{\infty}$ where each true arithmetical statement is provable. S. Feferman showed that an equivalent formal system $T h^{\#}$ can be obtained by erecting on $T h=P A$ a transfinite progression of formal systems $P A_{\infty}$ according to the following scheme

$$
\begin{aligned}
& P A_{0}=P A \\
& P A_{\alpha+1}=P A_{\alpha}+\left\{\forall x \operatorname{Pr}_{P A_{\alpha}}\left([A(\dot{x})]^{c}\right) \rightarrow \forall x A(x)\right\}, \\
& P A_{\lambda}=\bigcup_{\alpha<\lambda} P A_{\alpha}
\end{aligned}
$$

where $A(x)$ is a formula with one free variable and $\lambda$ is a limit ordinal. Then $T h=\bigcup_{\alpha \in O} P A_{\alpha}, O$ being Kleene's system of ordinal notations, is equivalent to $T h^{\#}=P A_{\infty}$. It is easy to see that $T h^{\#}=P A^{\#}$, i.e. $T h^{\#}$ is a maximally nice extension of the $\boldsymbol{P} \boldsymbol{A}$.

\section{Generalized Löb's Theorem}

Definition 3.1. An $T h-w f f \Phi$ (well-formed formula $\Phi$ ) is closed i.e., $\Phi$ is a $T h$-sentence iff it has no free variables; a wff $\Psi$ is open if it has free variables. We'll use the slang " $k$-place open wff" to mean a wff with $k$ distinct free variables. Given a model $M^{T h}$ of the $\boldsymbol{T h}$ and a $\boldsymbol{T h}$-sentence $\Phi$, we assume known the meaning of $M \vDash \Phi-i . e . \quad \Phi$ is true in $M^{T h}$, (see for example [4-6]).

Definition 3.2. Let $M_{\omega}^{T h}$ be an $\omega$-model of the $T h$. We shall say that, $T h^{\#}$ is a nice theory over $T h$ or a nice extension of the $T h$ iff:

1) $T h^{\#}$ contains $T h$;

2) Let $\Phi$ be any closed formula, then

$$
\left[T h \vdash \operatorname{Pr}_{T h}\left([\Phi]^{c}\right)\right] \&\left[M_{\omega}^{T h} \vDash \Phi\right]
$$

implies $T h^{\#} \vdash \Phi$.

Definition 3.3. We shall say that $T h^{\#}$ is a maximally nice theory over $T h$ or a maximally nice extension of the $T h$ iff $T h^{\#}$ is consistent and for any consistent nice extension $T h^{\prime}$ of the $T h: \operatorname{Ded}\left(T h^{\#}\right) \subseteq \operatorname{Ded}\left(T h^{\prime}\right)$ implies $\operatorname{Ded}\left(T h^{\#}\right)=\operatorname{Ded}\left(T h^{\prime}\right)$. 
Lemma 3.1. Assume that: 1) Con(Th); and 2) $T h \vdash \operatorname{Pr}_{T h}\left([\Phi]^{c}\right)$, where $\Phi$ is a closed formula. Then $T h \nvdash \operatorname{Pr}_{T h}\left([\neg \Phi]^{c}\right)$.

Proof. Let $\operatorname{Con}_{T h}(\Phi)$ be the formula

$$
\mathrm{Con}_{T h}(\Phi)
$$$$
\triangleq \forall t_{1} \forall t_{2} \neg\left[\operatorname{Prov}_{T h}\left(t_{1},[\Phi]^{c}\right) \wedge \operatorname{Prov}_{T h}\left(t_{2}, \operatorname{neg}\left([\Phi]^{c}\right)\right)\right]
$$$$
\leftrightarrow \neg \exists t_{1} \neg \exists t_{2}\left[\operatorname{Prov}_{T h}\left(t_{1},[\Phi]^{c}\right) \wedge \operatorname{Prov}_{T h}\left(t_{2}, \operatorname{neg}\left([\Phi]^{c}\right)\right)\right]
$$

where $t_{1}, t_{2}$ is a closed term. We note that under canonical observation, one obtains

$T h+\operatorname{Con}(T h) \vdash \operatorname{Con}_{T h}(\Phi)$ for any closed wff $\Phi$.

Suppose that $T h \vdash \operatorname{Pr}_{T h}\left([\neg \Phi]^{c}\right)$, then assumption (ii) gives

$$
T h \vdash \operatorname{Pr}_{T h}\left([\Phi]^{c}\right) \wedge \operatorname{Pr}_{T h}\left([\neg \Phi]^{c}\right) .
$$

From (3.1) and (3.2) one obtain

$$
\exists t_{1} \exists t_{2}\left[\operatorname{Prov}_{T h}\left(t_{1},[\Phi]^{c}\right) \wedge \operatorname{Prov}_{T h}\left(t_{2}, \operatorname{neg}\left([\Phi]^{c}\right)\right)\right] .
$$

But the Formula (3.3) contradicts the Formula (3.1). Therefore: $\operatorname{Th} \nvdash \operatorname{Pr}_{T h}\left([\neg \Phi]^{c}\right)$.

Lemma 3.2. Assume that: 1) $\operatorname{Con}(T h)$; and 2) $T h \vdash \operatorname{Pr}_{T h}\left([\neg \Phi]^{c}\right)$, where $\Phi$ is a closed formula. Then $T h \nvdash \operatorname{Pr}_{T h}\left([\Phi]^{c}\right)$.

Theorem 3.1. $[7,8]$. (Generalized Löb's Theorem). Assume that: $\operatorname{Con}(T h)$. Then theory $T h$ can be extended to a maximally consistent nice theory $T h^{\#}$ over $T h$.

Proof. Let $\Phi_{1} \cdots \Phi_{i} \cdots$ be an enumeration of all wff's of the theory $\boldsymbol{T h}$ (this can be achieved if the set of propositional variables can be enumerated). Define a chain $\wp=\left\{T h_{i} \mid i \in \mathbb{N}\right\}, T h_{1}=T h$ of consistent theories inductively as follows: assume that theory $T h_{i}$ is defined.

1) Suppose that a statement (3.4) is satisfied

$$
\begin{aligned}
& T h \vdash \operatorname{Pr}_{T h}\left(\left[\Phi_{i}\right]^{c}\right) \text { and } \\
& {\left[T h_{i} \nvdash \Phi_{i}\right] \&\left[M_{\omega}^{T h} \vDash \Phi_{i}\right]}
\end{aligned} .
$$

Then we define theory $T h_{i+1}$ as follows

$$
T h_{i+1} \triangleq T h_{i} \cup\left\{\Phi_{i}\right\} .
$$

2) Suppose that a statement (3.5) is satisfied

$$
\begin{aligned}
& T h \vdash \operatorname{Pr}_{T h}\left(\left[\neg \Phi_{i}\right]^{c}\right) \text { and } \\
& {\left[T h_{i} \nvdash \neg \Phi_{i}\right] \&\left[M_{\omega}^{T h} \vDash \neg \Phi_{i}\right]}
\end{aligned} .
$$

Then we define theory $T h_{i+1}$ as follows:

$$
T h_{i+1} \triangleq T h_{i} \cup\left\{\neg \Phi_{i}\right\} .
$$

3) Suppose that a statement (3.6) is satisfied

$$
T h \vdash \operatorname{Pr}_{T h}\left(\left[\Phi_{i}\right]^{c}\right) \text { and } T h_{i} \vdash \Phi_{i} .
$$

Then we define theory $T h_{i+1}$ as follows:

$$
T h_{i+1} \triangleq T h_{i} \cup\left\{\Phi_{i}\right\} .
$$

4) Suppose that a statement (3.7) is satisfied

$$
T h \vdash \operatorname{Pr}_{T h}\left(\left[\neg \Phi_{i}\right]^{c}\right) \text { and } T h \vdash \neg \Phi_{i} .
$$

Then we define theory $T h_{i+1}$ as follows:

$$
T h_{i+1} \triangleq T h_{i} .
$$

We define now theory $T h^{\#}$ as follows:

$$
T h^{\#} \triangleq \bigcup_{i \in \mathbb{N}} T h_{i} .
$$

First, notice that each $T h_{i}$ is consistent. This is done by induction on $i$ and by Lemmas 3.1-3.2. By assumption, the case is true when $i=1$. Now, suppose $T h_{i}$ is consistent. Then its deductive closure $\operatorname{Ded}\left(T h_{i}\right)^{i}$ is also consistent. If a statement (3.6) is satisfied i.e.,

$T h \vdash \operatorname{Pr}_{T h}\left(\left[\Phi_{i}\right]^{c}\right)$ and $T h \vdash \Phi_{i}$, then clearly

$T h_{i+1} \triangleq T h_{i} \cup\left\{\Phi_{i}\right\}$ is consistent since it is a subset of closure $\operatorname{Ded}\left(T h_{i}\right)$. If a statement (3.7) is satisfied, i.e., $T h \vdash \operatorname{Pr}_{T h}\left(\left[\neg \Phi_{i}\right]^{c}\right)$ and $T h_{i} \vdash \neg \Phi_{i}$, then clearly

$T h_{i+1} \triangleq T h_{i} \cup\left\{\neg \Phi_{i}\right\} \quad$ is consistent since it is a subset of closure $\operatorname{Ded}\left(T h_{i}\right)$.

Otherwise:

1) if a statement (3.4) is satisfied, i.e.

$T h_{i} \vdash \operatorname{Pr}_{\mathrm{Th}_{i}}\left(\left[\Phi_{i}\right]^{c}\right)$ and $T h_{i} \nvdash \Phi_{i}$, then clearly

$T h_{i+1} \triangleq T h_{i} \cup\left\{\Phi_{i}\right\}$ is consistent by Lemma 3.1 and by one of the standard properties of consistency: $\Delta \cup\{A\}$ is consistent iff $\Delta \nvdash \neg A$;

2) if a statement (3.5) is satisfied, i.e.

$T h \vdash \operatorname{Pr}_{T h}\left(\left[\neg \Phi_{i}\right]^{c}\right)$ and $T h_{i} \nvdash \neg \Phi_{i}$, then clearly

$T h_{i+1} \triangleq T h_{i} \cup\left\{\neg \Phi_{i}\right\} \quad$ is consistent by Lemma 3.2 and by one of the standard properties of consistency: $\Delta \bigcup\{\neg A\}$ is consistent iff $\Delta \nvdash A$.

Next, notice $\operatorname{Ded}\left(T h^{\#}\right)$ is a maximally consistent nice extension of the $\operatorname{set} \operatorname{Ded}(T h)$. A set $\operatorname{Ded}\left(T h^{\#}\right)$ is consistent because, by the standard Lemma 3.3 below, it is the union of a chain of consistent sets. To see that $\operatorname{Ded}\left(T h^{\#}\right)$ is maximal, pick any wff $\Phi$. Then $\Phi$ is some $\Phi_{i}$ in the enumerated list of all wff's. Therefore 
for any $\Phi$ such that $T h \vdash \operatorname{Pr}_{T h}\left([\Phi]^{c}\right)$ or $T h \vdash \operatorname{Pr}_{T h}\left([\neg \Phi]^{c}\right)$, either $\Phi \in T h^{\#}$ or $\neg \Phi \in T h^{\#}$.

Since $\operatorname{Ded}\left(T h_{i+1}\right) \subseteq \operatorname{Ded}\left(T h^{*}\right)$, we have $\Phi \in \operatorname{Ded}\left(T h^{\#}\right)$ or $\neg \Phi \in \operatorname{Ded}\left(T h^{\#}\right)$, which implies that $\operatorname{Ded}\left(T h^{\#}\right)$ is maximally consistent nice extension of the $\operatorname{Ded}(T h)$.

Lemma 3.3. The union of a chain $\wp=\left\{\Gamma_{i} \mid i \in \mathbb{N}\right\}$ of the consistent sets $\Gamma_{i}$, ordered by $\subseteq$, is consistent.

Definition 3.4. (a) Assume that a theory $T h$ has an $\omega$-model $M_{\omega}^{T h}$ and $\Phi$ is a $T h$-sentence. Let $\Phi_{\omega}$ be a $T h$-sentence $\Phi$ with all quantifiers relativised to $\omega$-model $M_{\omega}^{\text {Th }}$;

(b) Assume that a theory Th has a standard model $S M^{T h}$ and $\Phi$ is an $T h$-sentence. Let $\Phi_{S M}$ be a $T h$-sentence $\Phi$ with all quantifiers relativized to a model $S M^{T h}$ [9].

Remark 3.1. In some special cases we denote a sentence $\Phi_{\omega}$ by a symbol $\Phi\left[M_{\omega}^{T h}\right]$ and we denote a sentence $\Phi_{S M}$ by symbol $\Phi\left[M^{T h}\right]$ correspondingly.

Definition 3.5. (a) Assume that $T h$ has an $\omega$-model $M_{\omega}^{T h}$. Let $T h_{\omega}$ be a theory $T h$ relativized to a model $M_{\omega}^{T h}$, that is, any $T h_{\omega}$-sentence has a form $\Phi_{\omega}$ for some $T h$-sentence $\Phi$ [9];

(b) Assume that $T h$ has an standard model $S M^{T h}$. Let $T h_{S M}$ be a theory $T h$ relativized to a model $S M^{T h}$, that is, any $T h_{S M}$-sentence has a form $\Phi_{S M}$ for some $T h$ sentence $\Phi[9]$.

Remark 3.2. In some special cases we denote a theory $T h_{\omega}$ by symbol $T h\left[M_{\omega}^{T h}\right]$ and we denote a theory $T h_{S M}$ by symbol $T h\left[M^{T h}\right]$ correspondingly.

Theorem 3.2. (Strong Reflection Principle).

(i) Assume that: $T h$ has an $\omega$-model $M_{\omega}^{T h}$. Then for any $T h_{\omega}$-sentence $\Phi_{\omega}$

$$
T h_{\omega} \vdash \operatorname{Pr}_{T h_{\omega}}\left(\left[\Phi_{\omega}\right]^{c}\right) \text { iff } T h_{\omega} \vdash \Phi_{\omega} .
$$

(ii) Assume that: $T h$ has model $M_{S M}^{T h}$. Then for any $T h_{S M}$-sentence $\Phi_{S M}$

$$
T h_{S M} \vdash \operatorname{Pr} T h_{S M}\left(\left[\Phi_{S M}\right]^{c}\right) \text { iff } T h_{S M} \vdash \Phi_{S M} .
$$

Proof. (i) The one direction is obvious. For the other, assume that

$$
T h_{\omega} \vdash \operatorname{Pr}_{T h_{\omega}}\left(\left[\Phi_{\omega}\right]^{c}\right), T h_{\omega} \nvdash \Phi_{\omega},
$$

and $T h_{\omega} \vdash \neg \Phi_{\omega}$. Then

$$
T h_{\omega} \vdash \operatorname{Pr}_{T h_{\omega}}\left(\left[\neg \Phi_{\omega}\right]^{c}\right) .
$$

Note that $\operatorname{Con}\left(T h_{\omega}\right)$ holds since $\exists M_{\omega}^{T h}$. Let $\mathrm{Con}_{T h_{\omega}}$ be the formula

$$
\begin{aligned}
& \operatorname{Con}_{T h_{\omega}} \leftrightarrow \forall t_{1} \forall t_{2} \forall t_{3}\left(t_{3}=\left[\Phi_{\omega}\right]^{c}\right) \\
& \neg\left[\operatorname{Prov}_{T h_{\omega}}\left(t_{1},\left[\Phi_{\omega}\right]^{c}\right) \wedge \operatorname{Prov}_{T h_{\omega}}\left(t_{2}, \operatorname{neg}\left(\left[\Phi_{\omega}\right]^{c}\right)\right)\right] \\
& \leftrightarrow \neg \exists t_{1} \neg \exists t_{2} \neg \exists t_{3}\left(t_{3}=\left[\Phi_{\omega}\right]^{c}\right) \\
& \times\left[\operatorname{Prov}_{T h_{\omega}}\left(t_{1},\left[\Phi_{\omega}\right]^{c}\right) \wedge \operatorname{Prov}_{T h_{\omega}}\left(t_{2}, \operatorname{neg}\left(\left[\Phi_{\omega}\right]^{c}\right)\right)\right] .
\end{aligned}
$$

where $t_{1}, t_{2}, t_{3}$ is a closed term. Note that for any $\omega$ model $M_{\omega}^{T h}$ by the canonical observation one obtains the equivalence $\operatorname{Con}\left(T h_{\omega}\right) \leftrightarrow \operatorname{Con}_{T h_{\omega}}$ (see [2]). But the Formulae (3.11)-(3.12) contradicts the Formula (3.13). Therefore

$$
T h_{\omega} \nvdash \Phi_{\omega}, \nvdash \operatorname{Pr}_{T h_{\omega}}\left(\left[\neg \Phi_{\omega}\right]^{c}\right) \text { and } T h_{\omega} \nvdash \neg \Phi_{\omega} .
$$

Then theory $T h_{\omega}^{\prime}=T h_{\omega}+\neg \Phi_{\omega}$ is consistent and from the above observation one obtains that:

$\operatorname{Con}\left(T h_{\omega}^{\prime}\right) \leftrightarrow \operatorname{Con}_{T h_{\omega}^{\prime}}$, where

$$
\begin{aligned}
& \operatorname{Con}_{T h_{\omega}^{\prime}} \leftrightarrow \neg \exists t_{1} \neg \exists t_{2} \neg \exists t_{3}\left(t_{3}=\left[\Phi_{\omega}\right]^{c}\right) \\
& \times\left[\operatorname{Prov}_{T h_{\omega}^{\prime}}\left(t_{1},\left[\Phi_{\omega}\right]^{c}\right) \wedge \operatorname{Prov}_{T h_{\omega}^{\prime}}\left(t_{2}, \operatorname{neg}\left(\left[\Phi_{\omega}\right]^{c}\right)\right)\right] .
\end{aligned}
$$

On the other hand one obtains

$$
T h_{\omega}^{\prime} \vdash \operatorname{Pr}_{T h_{\omega}^{\prime}}\left(\left[\Phi_{\omega}\right]^{c}\right), T h_{\omega}^{\prime} \vdash \operatorname{Pr}_{T h_{\omega}^{\prime}}\left(\left[\neg \Phi_{\omega}\right]^{c}\right) .
$$

But the Formulae (3.15) contradicts the Formula (3.14). This contradiction completed the proof. Proof (ii) similarly as the proof (i) above.

\section{Definition 3.6.}

Let $T h$ be a theory such that the Assumption 1.1 is satisfied.

(a) Let $\Xi^{T h_{\omega}} \equiv \operatorname{Con}\left(T h ; M_{\omega}^{T h}\right)$ be a sentence in $T h$ asserting that $T h$ has $\omega$-model $M^{T h}$.

(b) Let $\Xi^{T h_{S M}} \equiv \operatorname{Con}\left(T h ; M_{S M}^{T h}\right)^{\rho}$ be a sentence in $T h$ asserting that $T h$ has standard model $M_{S M}^{T h}$.

Assumption 3.1. We assume that (i) a sentence $\Xi^{T h_{\omega}}$ is expressible in Th, i.e., a sentence $\Xi^{T h_{\omega}}$ is expressible by using the lenguage $\mathcal{L}_{T h}$ of the $T h$; (ii) a sentence $\Xi^{T h_{S M}}$ is expressible in Th, i.e., a sentence $\Xi^{T h_{S M}}$ is expressible by using the lenguage $\mathcal{L}_{T h}$ of the $T h$.

Remark 3.3. Note that (i) for any $\omega$-model $M_{\omega}^{T h}$ of the $T h$ by the canonical observation (see [2]) one obtains the equivalence

$$
\begin{aligned}
& \operatorname{Con}\left(T h ; M_{\omega}^{T h}\right) \leftrightarrow \\
& \operatorname{Con}\left(T h\left[M_{\omega}^{T h}\right]\right) \leftrightarrow \operatorname{Con}_{T h\left[M_{\omega}^{T h}\right]},
\end{aligned}
$$

(see remark 3.1) and the equivalence 


$$
\operatorname{Con}_{\operatorname{Th}\left[M_{\omega}^{T h}\right]} \leftrightarrow \neg \operatorname{Pr}_{T h\left[M_{\omega}^{T h}\right.}\left(\left[\digamma\left[M_{\omega}^{T h}\right]\right]^{c}\right)
$$

(see remark 3.2), where $\digamma$ is a closed formula refutable in $T h$.

(ii) for any standard model $M_{\omega}^{T h}$ of the $T h$ by the canonical observation (see [2] chapter), one obtains the equivalence

$$
\operatorname{Con}\left(T h ; M_{S M}^{T h}\right) \leftrightarrow \operatorname{Con}\left(T h\left[M_{S M}^{T h}\right]\right) \leftrightarrow \operatorname{Con}_{T h\left[M_{S M}^{T h}\right]}
$$

(see remark 3.1) and the equivalence

$$
\operatorname{Con}_{T h\left[M_{S M}^{T h}\right]} \leftrightarrow \neg \operatorname{Pr}_{T h_{S M}}\left(\left[\digamma\left[M_{S M}^{T h}\right]\right]^{c}\right) \square
$$

(see remark 3.2), where $\digamma$ is a closed formula refutable in $T h$.

Lemma 3.4. (I) Assume that $T h$ has $\omega$-model $M_{\omega}^{T h}$. Let $T h_{1}$ be a theory $T h_{1}=T h+\Xi^{T h_{\omega}}$. Then $T h_{1}$ is consistent.

(II) Assume that $T h$ has standard model $S M^{T h}$.

Let $T h_{2}$ be a theory $T h_{2}=T h+\Xi^{T h_{S M}}$. Then $T h_{2}$ is consistent.

Proof. (I) Assume that a theory

$T h_{1}=T h+\Xi^{T h_{\omega}} \equiv T h+\operatorname{Con}\left(T h ; M_{\omega}^{T h}\right)$ is inconsistent:

$\neg \operatorname{Con}\left(T h_{1}\right)$. This means that there is no any model $M^{T h}$ of $T h$ in which $\operatorname{Con}\left(T h ; M_{\omega}^{T h}\right)$ is true and in particular that is $T h$ has no any $\omega$-model $M_{1, \omega}^{T h}$ of $T h$ in which $\operatorname{Con}\left(T h ; M_{\omega}^{T h}\right)$ is true, i.e., $M_{1, \omega}^{T h} \not \models \Xi^{T h_{\omega}}\left[M_{1, \omega}^{T h}\right] \equiv \operatorname{Con}\left(T h ; M_{\omega}^{T h}\right)\left[M_{1, \omega}^{T h}\right]$ and therefore one obtains for any $\omega$-model $M_{1, \omega}^{T h}$ of $T h$ that

$$
M_{1, \omega}^{T h} \vDash \neg \operatorname{Con}\left(T h ; M_{\omega}^{T h}\right)\left[M_{1, \omega}^{T h}\right],
$$

and in particular

$$
M_{1, \omega}^{T h} \vDash \neg \operatorname{Con}\left(T h ; M_{1, \omega}^{T h}\right)\left[M_{1, \omega}^{T h}\right],
$$

From (3.21) using (3.16)-(3.17) and one obtains

$M_{1, \omega}^{T h} \vDash \neg \operatorname{Con}_{T h\left[M_{1, \omega}^{T h}\right]}\left[M_{1, \omega}^{T h}\right] \leftrightarrow \operatorname{Pr}_{\operatorname{Th}\left[M_{1, \omega}^{T h}\right]}\left(\left[\digamma\left[M_{1, \omega}^{T h}\right]\right]^{c}\right)$.

From (3.22) and Theorem 3.2(I) one obtains

$$
M_{1, \omega}^{T h} \vDash\left(\left[\digamma\left[M_{1, \omega}^{T h}\right]\right]^{c}\right) .
$$

Obviously (3.23) contradicts to the assumption that $T h$ has an $\omega$-model $M_{\omega}^{T h}$. This contradiction completed the proof.

Theorem 3.3. (I) $T h$ has no any $\omega$-model $M_{\omega}^{T h}$.

(II) $T h$ has no any standard model $S M^{T h}$.

Proof. (I) By Lemma 3.4(I) one obtains that $T h_{1} \vdash \operatorname{Con}\left(T h_{1}\right)$. But Godel's Second Incompleteness Theorem applied to $T h_{1}$ asserts that $\operatorname{Con}\left(T h_{1}\right)$ is unprovable in $T h_{1}$. This contradiction completed the proof.
Proof. (II) Similarly as above.

Remark 3.4. We emphasize that it is well known that axiom $\exists S M^{Z F C}$ a single statement in $Z F C$ see [10], Ch. II, section 7. We denote this statement through all this paper by symbol $\operatorname{Con}\left(Z F C ; S M^{Z F C}\right)$.

Theorem 3.4. $Z F C$ has no any $\omega$-model $M_{\omega}^{Z F C}$.

Proof. Immediately follows from Theorem 3.3 (I) and Remark 3.4.

Theorem 3.5. $Z F C$ has no any standard model. $S M^{Z F C}$.

Proof. Immediately follows from Theorem 3.3 (II) and Remark 3.4.

Theorem 3.6. $Z F C$ is incompatible with all the usual large cardinal axioms [11] which imply the existence standard model of $Z F C$.

Proof. Theorem 3.6 immediately follows from Theorem 3.5.

Theorem 3.7. Let $\kappa$ be an inaccessible cardinal. Then $\neg \operatorname{Con}(Z F C+\exists \kappa)$.

Proof. Let $H_{\kappa}$ be a set of all sets having hereditary size less then $\kappa$. It easy to see that $H_{\kappa}$ forms standard model of $Z F C$. Therefore Theorem 3.7 immediately follows from Theorem 3.5.

\section{Conclusion}

In this paper we proved so-called strong reflection principles corresponding to formal theories $T h$ which has $\omega$-models $M_{\omega}^{T h}$ and in particular to formal theories $T h$, which has a standard models $S M^{T h}$. The assumption that there exists a standard model of $T h$ is stronger than the assumption that there exists a model of Th. This paper examined some specified classes of the standard models of $Z F C$ so-called strong standard models of $Z F C$. Such models correspond to large cardinals axioms. In particular we proved that theory $Z F C+\operatorname{Con}(Z F C)$ is incompatible with existence of any inaccessible cardinal $\kappa$. Note that the statement: Con $(Z F C+\exists$ some inaccessible cardinal $\kappa$ ) is $\Pi_{1}^{0}$. Thus Theorem 3.6 asserts there exist numerical counterexample which would imply that a specific polynomial equation has at least one integer root.

\section{REFERENCES}

[1] M. H. Löb, "Solution of a Problem of Leon Henkin," The Journal of Symbolic Logic, Vol. 20, No. 2, 1955, pp. 115-118. doi:10.2307/2266895

[2] C. Smorynski, "Handbook of Mathematical Logic," North-Holland Publishing Company, 1977.

[3] T. Drucker, "Perspectives on the History of Mathematical Logic," Birkhauser, Boston, 2008, p. 191.

[4] A. Marcja and C. Toffalori, "A Guide to Classical and Modern Model Theory (Series: Trends in Logic)," Springer, Berlin, 2003, p. 371. 
[5] F. W. Lawvere, C. Maurer and G. C. Wraith, "Model Theory and Topoi," Springer, Berlin, 1975.

[6] D. Marker, "Model Theory: An Introduction (Graduate Texts in Mathematics)," Springer, Berlin, 2002.

[7] J. Foukzon, "Generalized Löb's Theorem," 2013. http://arxiv.org/abs/1301.5340

[8] J. Foukzon, "An Possible Generalization of the Löb's Theorem," AMS Sectional Meeting AMS Special Session. Spring Western Sectional Meeting University of Colorado Boulder, Boulder, 13-14 April 2013.

http://www.ams.org/amsmtgs/2210_abstracts/1089-03-60. pdf

[9] P. Lindstrom, "First Order Predicate Logic with Generalized Quantifiers," Theoria, Vol. 32, No. 3, 1966, pp. 186-195.

[10] P. Cohen, "Set theory and the continuum hypothesis," Reprint of the W. A. Benjamin, Inc., New York, 1966 edition; 1966. ISBN-13: 978-0486469218

[11] A. Kanamori, "The Higher Infinite: Large Cardinals in Set Theory from Their Beginnings," 2nd Edition, Springer, Berlin, 2003. 